Article

\title{
Synthesis and Characterization of "Ravine-Like" BCN Compounds with High Capacitance
}

\author{
Dongping Chen, Yanzhen Huang, Xinling Hu, Rongkai Li, Yingjiang Qian and Dongxu Li * (D) \\ College of Materials Science and Engineering, Huaqiao University, Xiamen 361021, China; \\ 1511302040@hqu.edu.cn (D.C.); 15737567342@163.com (Y.H.); 18224566342@163.com (X.H.); \\ liyungkai@yeah.net (R.L.); qian1358_love@163.com (Y.Q.) \\ * Correspondence: lidongxu@hqu.edu.cn; Tel.: +86-130-5551-1552
}

Received: 22 December 2017; Accepted: 26 January 2018; Published: 29 January 2018

\begin{abstract}
A series of "ravine-like" boron carbonitrides (abbreviation: $\mathrm{BCN}$ ) were synthesized by a green precursor pyrolysis method at different temperatures (about $700-1100{ }^{\circ} \mathrm{C}$ ). The highest electrochemical performance of $\mathrm{BCN}-800$ (Named $\mathrm{BCN}$-temperature) electrode was observed, because the "ravine-like" structure can significantly increase the contact area and improve the wettability between electrode and electrolyte. The BCN electrode exhibited ultrahigh specific capacitance $805.9 \mathrm{~F} / \mathrm{g}$ (at a current density of $0.2 \mathrm{~A} / \mathrm{g}$ ), excellent rate capability, and good cycling stability $(91 \%)$ after 3000 cycles at a current density of $8 \mathrm{~A} / \mathrm{g}$, showing high potential applications in supercapacitors.
\end{abstract}

Keywords: pyrolysis; boron carbonitride; electrochemical performance; supercapacitors

\section{Introduction}

In recent years, two-dimensional (2D) materials have attracted considerable attention because of their unique physical and chemical properties, which ensure excellent performance in many fields, such as catalysts, semiconductors, energy storage, and electronics [1-3]. Among these 2D materials, boron carbonitride $(\mathrm{BCN})$ has attracted the interest of physical, chemical, and materials science researchers. Numerous theoretical and experimental studies have shown that $\mathrm{BCN}$ compounds exhibited various potential applications because of their excellent electrical, optical, thermal, and mechanical properties, which are attributed to their similar structures to $\mathrm{BN}$ and $\mathrm{C}$ and their adjustable physical and chemical properties [4-6]. At present, most of the $\mathrm{BCN}$ prepared in experiments belong to $\mathrm{C}$-rich $\mathrm{BCN}$ compounds. Moreover, many preparation methods were presented, including magnetron sputtering, chemical vapor deposition, physical vapor deposition, and high-temperature and high-pressure (HTHP) methods. Liu et al. [7] successfully used the HTHP method to synthesize nearly-transparent $\mathrm{BCN}$ diamonds. Ma et al. [8] used the method of "thermal substitution" to dope $\mathrm{C}$ into $\mathrm{BN}$ to prepare $\mathrm{BCN}$ nanosheets which had a controllable band-gap and exhibited excellent nonlinear optical performance. Karbhal et al. [9] used the thermal decomposition method to synthesize $\mathrm{BCN}$ nanosheets with a high specific capacitance $(244 \mathrm{~F} / \mathrm{g})$. Finding and exploring new domains of applications of $\mathrm{BCN}$ compounds are hot spots in the field of materials science research.

Energy, the material basis of human activities, has been the focus of attention around the world. Supercapacitors exhibit excellent performance with high specific capacity, high energy density, high power, and long life cycle [10]. Thus, electric cars, mobile communications, defense science and technology, and many other fields have broad prospects (i.e., real green energy) [11]. In recent years, research on the use of $\mathrm{BCN}$ materials in supercapacitors has often been reported. Research shows that boron and nitrogen co-doping in carbon material effectively changes the electron donor/acceptor characteristics, which improves the capacitance performance of carbon materials [12].

This work presented a pyrolysis method to produce "ravine-like" BCN compounds at different temperatures. A certain amount of boric acid was mixed with 2,4,6-tri(2-pyridyl)-1,3,5-triazine 
to form the precursor, and the precursor was pyrolyzed to prepare $\mathrm{BCN}$ compounds in nitrogen atmosphere. Capacitance performance tests show that the specific capacitance of the "ravine-like" $\mathrm{BCN}$ compounds was 2-3 times higher than that reported in the literature under the same test conditions, which exhibits ultrahigh specific capacitance and excellent rate capability and has potential applications in supercapacitors.

\section{Results and Discussion}

The Fourier transform infrared (FTIR) spectra of BCN samples were collected to investigate the common features of as-prepared BCN samples under different temperatures (Figure 1). The absorption peaks at 792 and $1386 \mathrm{~cm}^{-1}$ are attributed to the bending vibration of out-of-plane B-N-B and transverse stretching vibration of in-plane $\mathrm{B}-\mathrm{N}$ bonds in all samples $[13,14]$. The small absorption peaks at 1086 and $1613 \mathrm{~cm}^{-1}$ correspond to the $\mathrm{B}-\mathrm{C}$ vibrations and $s p^{2} \mathrm{C}-\mathrm{N}$ bonds $[15,16]$. Another broad peak at $3417 \mathrm{~cm}^{-1}$ is attributed to the $\mathrm{O}-\mathrm{H}$ stretching vibration mode [17]. Regularly, intensities of $\mathrm{B}-\mathrm{N}$ and $\mathrm{B}-\mathrm{N}-\mathrm{B}$ peak increased with increasing pyrolysis temperature, whereas the $\mathrm{B}-\mathrm{C}$ and $\mathrm{C}-\mathrm{N}$ vibration modes decreased sharply. Absorption peaks of precursor appeared at $600{ }^{\circ} \mathrm{C}$, meaning that the precursor is still included. The FTIR results confirm the formation of atomic-level ternary $\mathrm{BCN}$ hybrid structures.

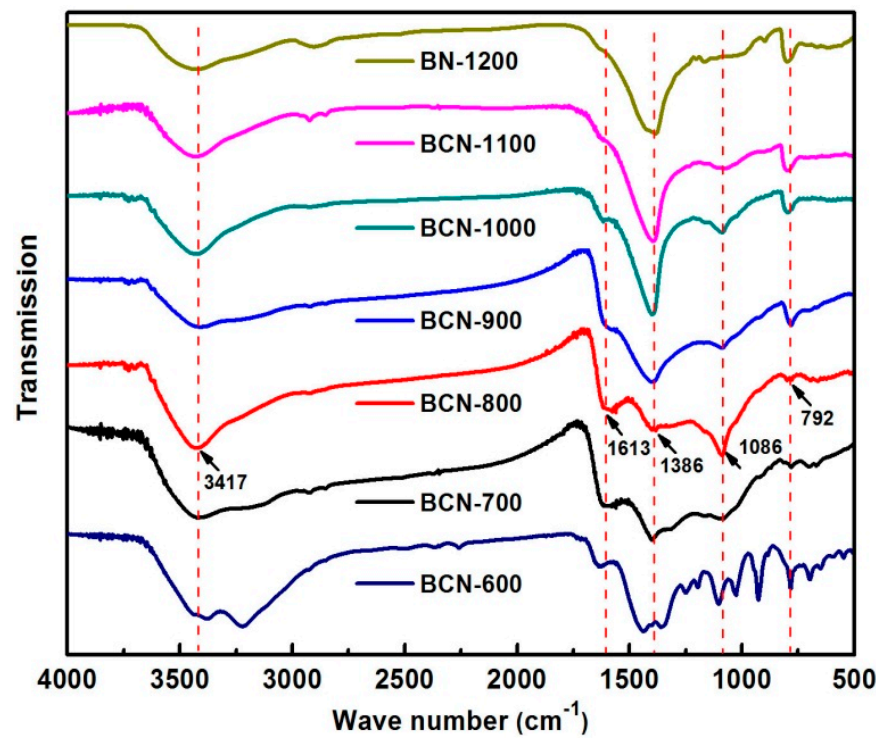

Figure 1. Fourier transform infrared (FTIR) spectra of boron carbonitride (BCN)-600, BCN-700, BCN-800, BCN-900, BCN-1000, BCN-1100, and BN-1200.

Figure 2 shows the $\mathrm{XRD}$ patterns of $\mathrm{BCN}$ samples obtained at different pyrolysis temperatures, which have two main broad reflections centered at $26.2^{\circ}$ and $43.6^{\circ}$, attributed to the (002) and (100) planes, respectively $[18,19]$. Furthermore, the XRD patterns at relatively low pyrolysis temperatures $\left(700{ }^{\circ} \mathrm{C}\right.$ to $900{ }^{\circ} \mathrm{C}$ ) are significantly broader than that of the other samples, which indicates the presence of the amorphous phase, composed of typical graphite-like BCN materials. With increase of pyrolysis temperature, the diffraction peak became sharper, indicating better crystallization. At $1100^{\circ} \mathrm{C}$, the product began to phase separate and $\mathrm{BN}$ was included mainly at $1200^{\circ} \mathrm{C}$. Obviously, unreacted precursor existed in BCN-600, in good agreement with FTIR results. Additionally, phase separation was observed over $1200^{\circ} \mathrm{C}$.

SEM was employed to investigate the morphology of the samples. As shown in Figure 3, "ravine-like" BCN compounds can be observed, with sizes of 150 to $200 \mathrm{~nm}$. However, the morphology of the "ravine-like" BCN was destroyed as temperature increased, and basically disappeared over $1200{ }^{\circ} \mathrm{C}$. TEM and corresponding HRTEM images were collected to analyze the microstructure of 
samples. The "ravine-like" structures of BCN-800 and BCN-900 were presented in HRTEM images (Figure 4). The lattice spacing was calculated to be $0.36 \mathrm{~nm}$, matching the (002) plane of samples in XRD. Meanwhile, with the increase of temperature, the crystal density of the samples increased and the crystal became larger (as seen in the Supplementary Figure S1A(d)). These conclusions are consistent with the results of XRD characterization. In addition, with the increase in pyrolysis temperature, the "ravine-like" morphology was obviously reduced, which might be attributed to disordered crystallization filling in the ravine.

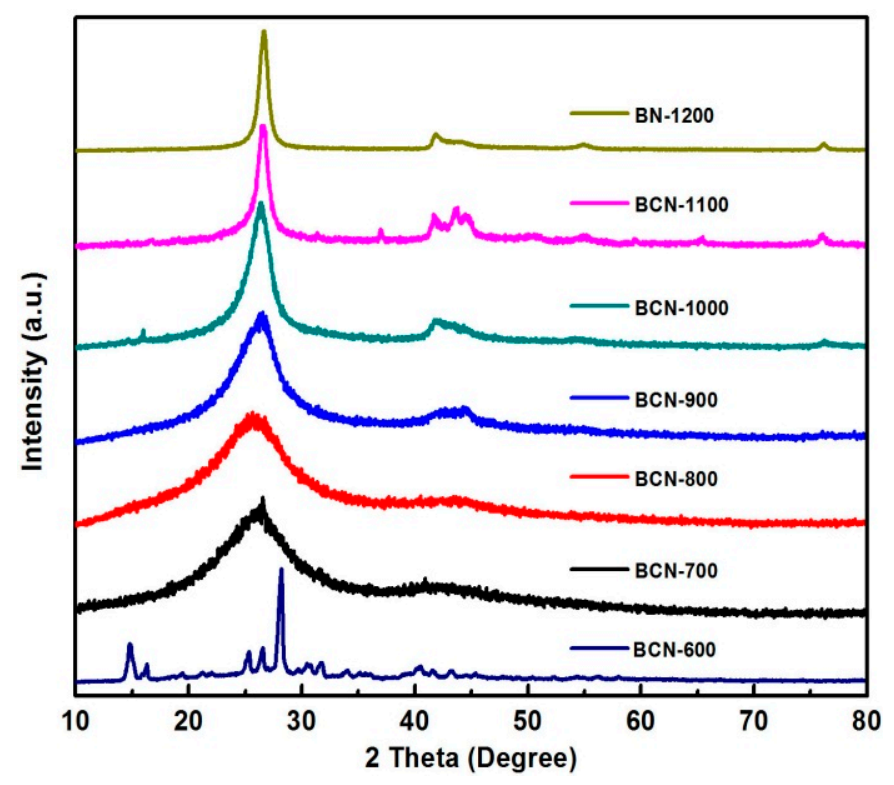

Figure 2. XRD pattern of BCN-600, BCN-700, BCN-800, BCN-900, BCN-1000, BCN-1100, and BN-1200.

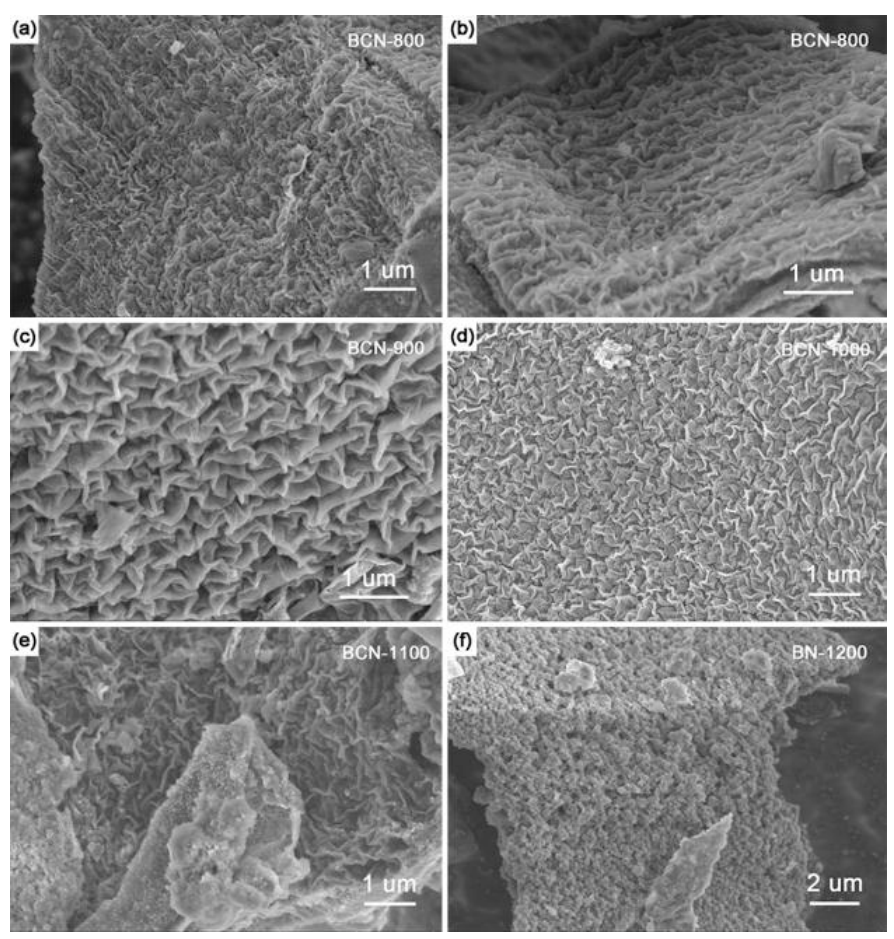

Figure 3. SEM images of (a,b) BCN-800; (c) BCN-900; (d) BCN-1000; (e) BCN-1100; (f) BN-1200. 


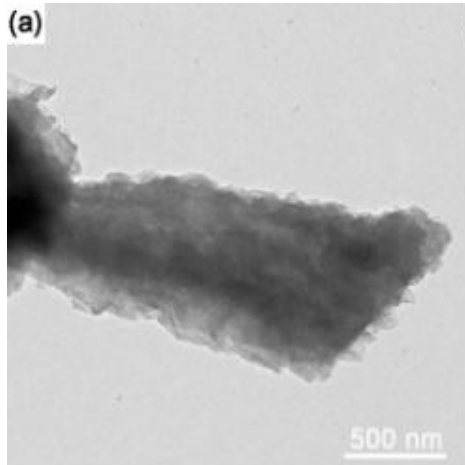

(d)

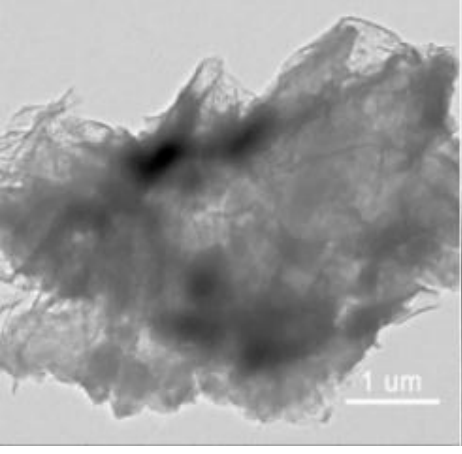

(b)
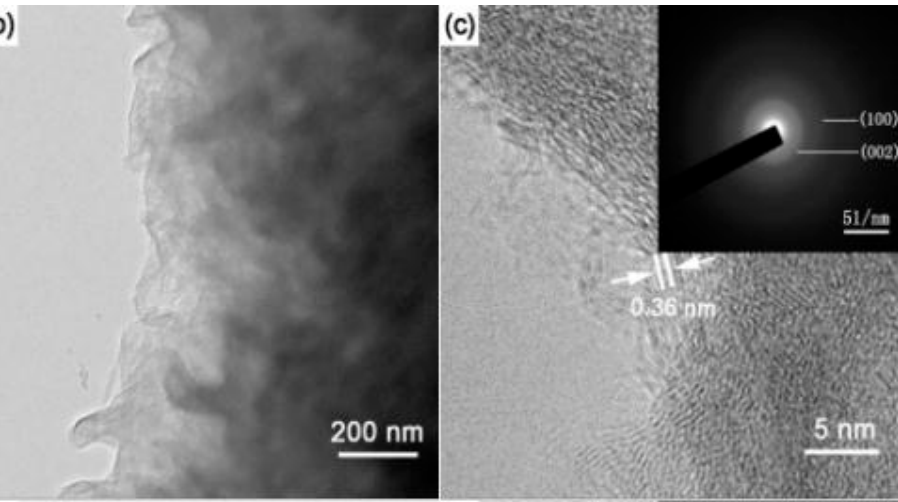

(e)

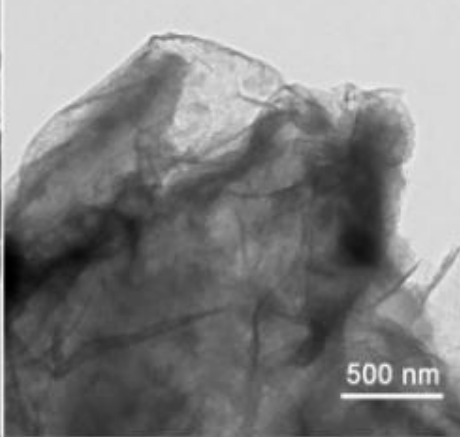

(f)

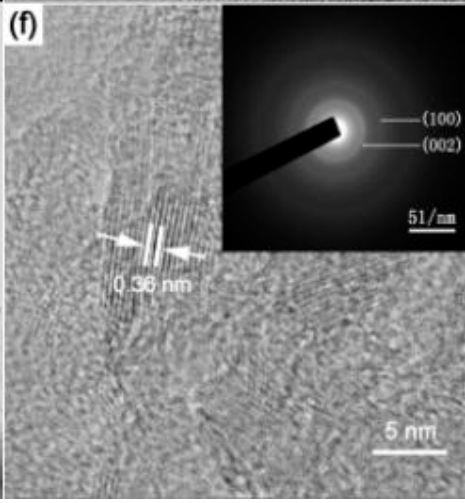

Figure 4. $(\mathbf{a}-\mathbf{c})$ TEM images and the corresponding HRTEM images of BCN-800; (d-f) TEM images and the corresponding HRTEM images of BCN-900.

For further characterization of the elemental composition, X-ray photoelectron spectroscopy (XPS) experiments were performed (Table 1 and Figure 5). The elemental compositions of BCN-800 were calculated to be $10.15,62.66,12.17$, and 15.02 at $\%$ for $\mathrm{B}, \mathrm{C}, \mathrm{N}$, and $\mathrm{O}$, respectively. With the increase of temperature, the contents of $\mathrm{B}$ and $\mathrm{N}$ increased. When the temperature increased to $1200{ }^{\circ} \mathrm{C}$, boron nitride (BN) was the main composition, which is consistent with the above results. In addition, the $\mathrm{O}$ 1s signal could be ascribed to moisture and other gases adsorbed on the surface because of the strongly oxophilic nature of B in the sample. Figure $5 \mathrm{~b}-\mathrm{d}$ shows the spectra of B 1s, C 1s, and N 1s. In the B 1s XPS spectrum (Figure 5b), two peaks centered at 191.3 and $192.4 \mathrm{eV}$ are identified, which can be attributed to the $\mathrm{B}-\mathrm{C}$ and $\mathrm{B}-\mathrm{N}$ bonding structures [20]. The $\mathrm{C} 1 \mathrm{~s}$ signal (Figure $5 \mathrm{c}$ ) could be ascribed to four peaks at approximately $284.5,285.1,286.6$, and $288.5 \mathrm{eV}$, corresponding to the $\mathrm{C}-\mathrm{B}, \mathrm{C}-\mathrm{C}, \mathrm{C}-\mathrm{N}$, and $\mathrm{C}-\mathrm{O}$ bonds, respectively [21]. The high-resolution $\mathrm{N} 1 \mathrm{~s}$ spectrum (Figure $5 \mathrm{~d}$ ) can be deconvoluted into three peaks at $398.7,399.8,401.2 \mathrm{eV}$, which are assigned to the $\mathrm{N}-\mathrm{B}$, graphitic $\mathrm{N}-\mathrm{C}$, and $\mathrm{N}=\mathrm{C}$ bonds, respectively [22].

Table 1. Quantitative elemental compositions of the samples derived from XPS surveys.

\begin{tabular}{ccccc}
\hline Sample & B (at \%) & C (at \%) & N (at \%) & O (at \%) \\
\hline BCN-700 & 13.02 & 52.54 & 13.83 & 20.61 \\
BCN-800 & 10.15 & 62.6 & 12.17 & 15.02 \\
BCN-900 & 20.94 & 48.7 & 16.23 & 14.11 \\
BCN-1000 & 43.09 & 14.0 & 32.9 & 9.97 \\
BCN-1100 & 40.87 & 14.9 & 30.9 & 13.1 \\
BN-1200 & 49.05 & 4.37 & 39.5 & 7.01 \\
\hline
\end{tabular}



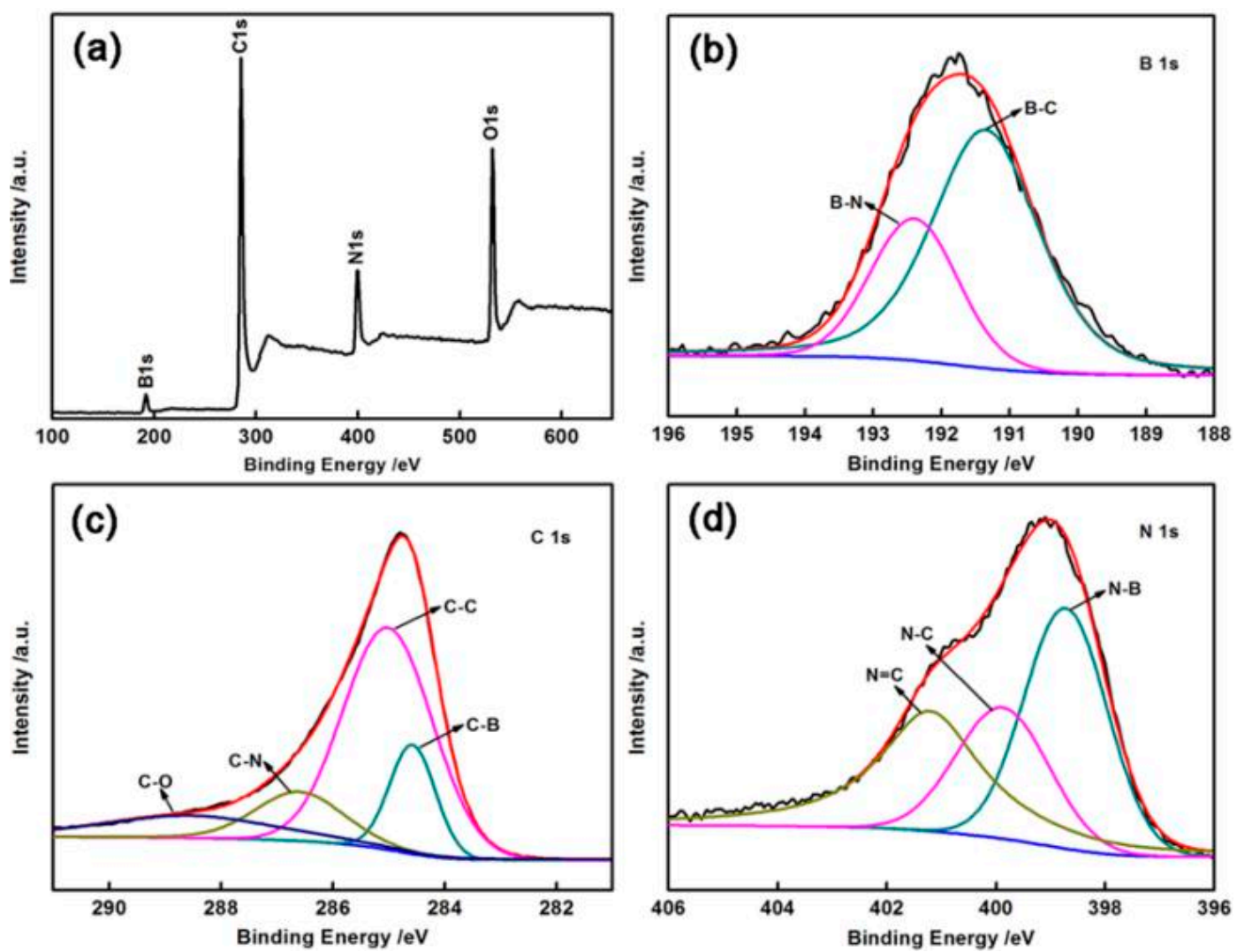

Figure 5. (a) The survey scan of X-ray photoelectron spectroscopy (XPS) on BCN-800; (b) B 1s XPS peak; (c) C 1s XPS peak and (d) N 1s XPS peak.

Cyclic voltammetry $(\mathrm{CV})$ was employed with $6.0 \mathrm{M} \mathrm{KOH}$ as electrolyte in a three-electrode system and a potential interval between $-1 \mathrm{~V}$ and $-0.2 \mathrm{~V}$ to investigate the electrochemical behavior of the as-prepared samples. Figure 6a shows the $\mathrm{CV}$ curves of the $\mathrm{BCN}-800$ electrode at different scan rates of $10,30,50,100$, and $200 \mathrm{mV} / \mathrm{s}$ in $6.0 \mathrm{M} \mathrm{KOH}$ solution. As illustrated in Figure $6 \mathrm{a}$, the curves exhibited a typical rectangular-like shape without a redox peak existing in the sample, which indicated the capacitive response from the electrical double-layer capacitor. The comparison of the curves shows that the enclosed area of the CV curve decreased drastically with the increase in temperature, which indicates that lowering the reaction temperature might enhance the curve area (as seen in the Supplementary Figure S9(a)).

Furthermore, galvanostatic charge/discharge experiments were performed to calculate the specific capacitance. Figure $6 \mathrm{~b}$ shows the galvanostatic charge/discharge of $\mathrm{BCN}-800$ with different current densities in $6.0 \mathrm{M} \mathrm{KOH}$ solution. According to the formula $I t / E$ (where $t$ is the discharge time, $I$ is the charge/discharge current, and $E$ is the voltage difference), the specific capacitances of BCN-700, BCN-800, BCN-900, BCN-1000, BCN-1100, and BN-1200 were $615.5 \mathrm{~F} / \mathrm{g}, 805.9 \mathrm{~F} / \mathrm{g}, 288.3 \mathrm{~F} / \mathrm{g}, 221.6 \mathrm{~F} / \mathrm{g}$, $173.2 \mathrm{~F} / \mathrm{g}, 14.4 \mathrm{~F} / \mathrm{g}$ at a current density of $0.2 \mathrm{~A} / \mathrm{g}$, respectively. Among them, $\mathrm{BCN}-800$ showed the largest specific capacitance. At the same current density, the specific capacitance of $\mathrm{BCN}-800$ was significantly higher than that of VA-BC ${ }_{2}$ NNTAs $(547 \mathrm{~F} / \mathrm{g})$ and VA-BCN $(321 \mathrm{~F} / \mathrm{g})$ [23-25]. The primary reason for this result was influenced by the morphology. Previous SEM and TEM tests showed that the morphology of BCN-800 was "ravine-like", which increases the contact area between electrode and electrolyte, thereby increasing the ion exchange between electrode and electrolyte and improving the specific capacitance of the BCN-800 electrode. 

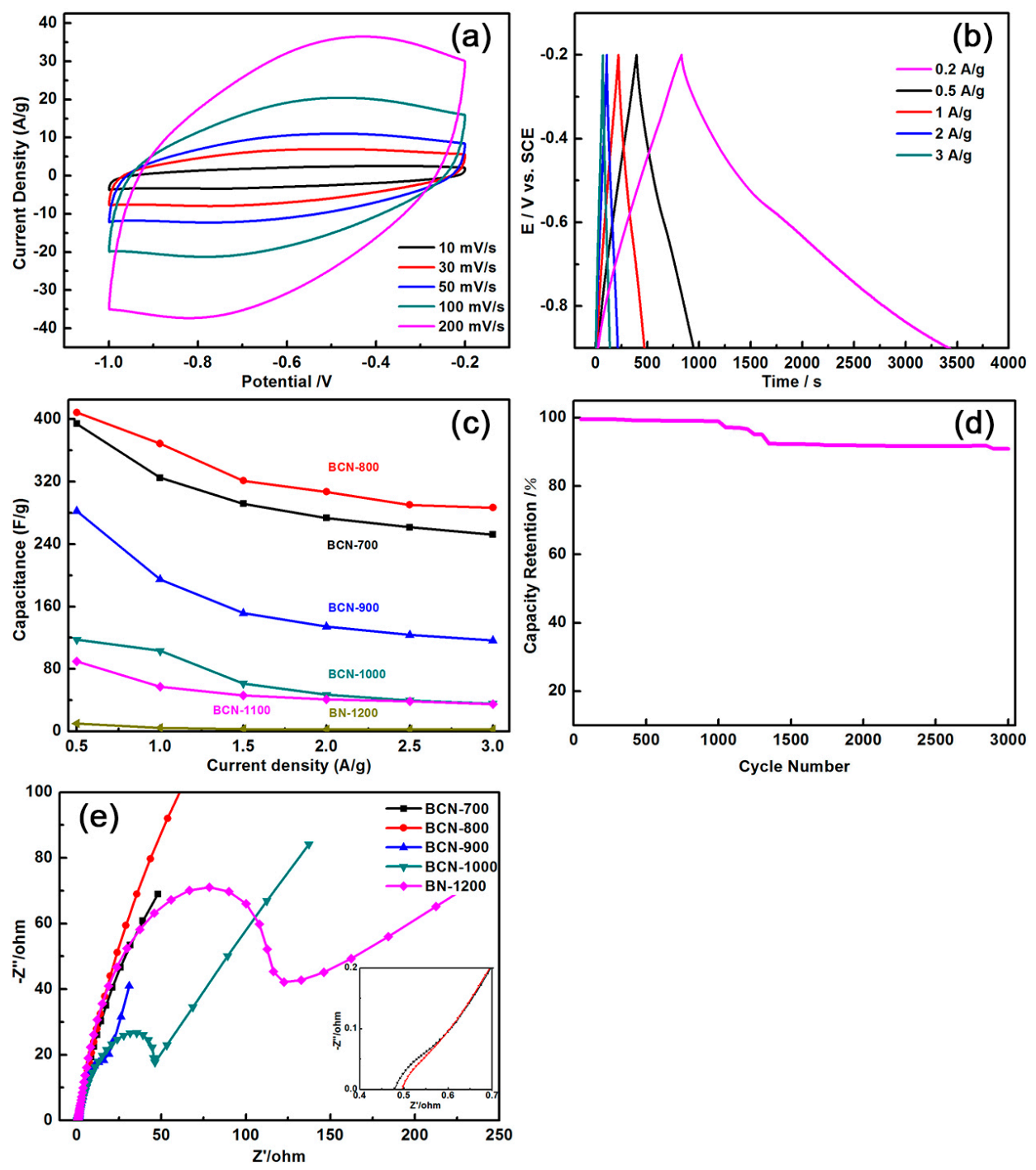

Figure 6. (a) Cyclic voltammetry (CV) curves of $\mathrm{BCN}-800$ at various scan rates in $6.0 \mathrm{M} \mathrm{KOH}$ electrolyte solution; (b) Galvanostatic charge/discharge curves of BCN-800 at a current density of $0.2 \mathrm{~A} / \mathrm{g}$ in $6.0 \mathrm{M}$ $\mathrm{KOH}$ electrolyte solution; (c) The specific capacitance at the current density from 0.5 to $3 \mathrm{~A} / \mathrm{g}$; (d) the stability evaluation of $\mathrm{BCN}-800$ electrode material in $6 \mathrm{M} \mathrm{KOH}$ solution at a charge current of $8 \mathrm{~A} / \mathrm{g}$; (e) Nyquist plots of samples in $6 \mathrm{M} \mathrm{KOH}$ solution. The inset shows the enlarged Nyquist plots in the high-frequency area of BCN-700 and BCN-800.

For an ideal supercapacitor, it is important to explore whether it can guarantee the same energy and good cycle stability under different operating conditions. Thus, this study investigated the capacitance retention rates of different samples. Figure $6 \mathrm{c}$ shows the specific capacitance obtained at current densities of $0.5,1,1.5,2,2.5$, and $3 \mathrm{~A} / \mathrm{g}$ to illustrate the rate capability. For the BCN-800 electrode, when the discharge current increased from $0.5 \mathrm{~A} / \mathrm{g}$ to $3 \mathrm{~A} / \mathrm{g}$, the specific capacitance could still be maintained at 70.18\%. However, for the BCN-700, BCN-900, BCN-1000, BCN-1100, and BN-1200 electrodes, the capacitance retention rates were only approximately $64.00 \%, 68.00 \%, 45.75 \%, 39.78 \%$, and $24.51 \%$, respectively. The durability of the BCN-800 electrode was examined by the continuous charge/discharge test at a current density of $8 \mathrm{~A} / \mathrm{g}$. Figure $6 \mathrm{~d}$ shows that capacitance could still be maintained at $91 \%$ after 3000 cycles, which indicates that the BCN-800 electrode had good stability and high rate capability. 
Electrochemical impedance spectroscopy (EIS) was studied for samples. The Nyquist plots are shown in Figure 6e, the semicircles of BCN-800 and BCN-700 are both smaller in the high-frequency range, indicating that both samples had smaller charge transfer resistance. However, the charge transfer resistance of $\mathrm{BCN}-700$ was greater than that of $\mathrm{BCN}-800$ (inset). With the increase of pyrolysis temperature, the semicircle of samples in the high-frequency range showed a significant increase of charge transfer resistance. The BCN-800 had the largest straight-line slope in the low-frequency region, indicating that it had the smallest electrolyte diffusion resistance and the best capacitance behavior. From the EIS studies, it can be concluded that $\mathrm{BCN}-800$ showed significant capacitive behavior and lower electrochemical charge transfer resistance. This conclusion is consistent with the results of electrochemical specific capacitance test.

\section{Materials and Methods}

\subsection{Preparation of the Precursor}

The "ravine-like" BCN compounds were synthesized via precursor pyrolysis. Firstly, boric acid $(0.886 \mathrm{~g})$ was thoroughly mixed with 2,4,6-tri(2-pyridyl)-1,3,5-triazine $(0.5 \mathrm{~g})$. Then, the mixture was dissolved in $20 \mathrm{~mL}$ of acetonitrile. The solution was kept in a $250 \mathrm{~mL}$ round-bottom flask with stirring, followed by refluxing at $70{ }^{\circ} \mathrm{C}$ for $8 \mathrm{~h}$. The precursor was collected after vacuum filtration and further drying overnight at $80^{\circ} \mathrm{C}$.

\subsection{Synthesis of "Ravine-Like" BCN Compounds}

The as-prepared precursor was placed in a corundum boat. Then, the boat was placed in a tube furnace and subsequently purged under nitrogen. The constant nitrogen flow was maintained throughout the entire procedure to obtain an inert atmosphere. The boat was held at $600{ }^{\circ} \mathrm{C}, 700{ }^{\circ} \mathrm{C}$, $800{ }^{\circ} \mathrm{C}, 900{ }^{\circ} \mathrm{C}, 1000{ }^{\circ} \mathrm{C}, 1100{ }^{\circ} \mathrm{C}$, and $1200^{\circ} \mathrm{C}$ for $3 \mathrm{~h}$ with a heating rate of $10^{\circ} \mathrm{C} / \mathrm{min}$, respectively. The final products (named $\mathrm{BCN}$-temperature) were obtained after washing with deionized water and drying overnight.

\subsection{Characterization}

Fourier transform infrared (FTIR) spectroscopy was conducted using the Nicolet iS50 to characterize the chemical structure. X-ray diffraction (XRD) patterns were observed using the Rigaku MiniFlex 600 (SCINCO CHINA, Shanghai, China) with $\mathrm{Cu}-\mathrm{K} \alpha$ radiation to analyze the crystal structure. Scanning electron microscopy (SEM) images were obtained using the Hitachi field emission scanning electron microscope, and transmission electron microscopy (TEM) images were collected through the JEM-2100 to examine the morphology and structure of the samples. X-ray photoelectron spectroscopy (XPS) was conducted using the Thermo ESCALAB 250 (Thermo Fisher Scientific, Shanghai, China) with an X-ray $\mathrm{Al} \mathrm{K} \alpha$ source to analyze the bonding state.

\section{Conclusions}

In summary, a facile synthesis method was presented to fabricate a series of "ravine-like" ternary $\mathrm{BCN}$ compounds. The method has the characteristics of brief reaction steps, high yield, and low cost. The range for synthesized $\mathrm{BCN}$ compounds was 700 to $1100{ }^{\circ} \mathrm{C}$. The BCN-800 electrode exhibited the highest specific capacitance, which is $805.9 \mathrm{~F} / \mathrm{g}$ at a current density of $0.2 \mathrm{~A} / \mathrm{g}$. This finding can be attributed to the "ravine-like" morphology of $\mathrm{BCN}-800$ that increases the contact area between electrode and electrolyte and facilitates ion exchange. In addition, the BCN-800 electrode presented excellent rate capability and good cycling stability (91\%) after 3000 cycles at a current density of $8 \mathrm{~A} / \mathrm{g}$. This excellent performance suggests high potential applications of BCN material in supercapacitors.

Supplementary Materials: The following are available online at http:/ /www.mdpi.com/1996-1944/11/02/209/ s1, Figure S1: TEM images and the corresponding HRTEM images of BCN-1000; Figure S1A: TEM images and the corresponding HRTEM images of BCN-1100; Figure S1B: TEM images and the corresponding HRTEM images 
of BN-1200; Figure S2: EDS and Elemental Mapping of BCN-800; Figure S2A: EDS and Elemental Mapping of BCN-1000; Figure S2B. EDS and Elemental Mapping of BN-1200; Figure S3. Adsorption and desorption curve of samples; (a) BCN-800; (b) BCN-900; (c) BCN-1000; (d) BCN-1100; (e) BN-1200; Figure S4. (a) The survey scan of XPS on BCN-700; (b) B 1s XPS peak; (c) C 1s XPS peak and (d) N 1s XPS peak; Figure S5. (a) The survey scan of XPS on BCN-900; (b) B 1s XPS peak; (c) C 1s XPS peak and (d) N 1s XPS peak; Figure S6. (a) The survey scan of XPS on BCN-1000; (b) B 1s XPS peak; (c) C 1s XPS peak and (d) N 1s XPS peak; Figure S7. (a) The survey scan of XPS on BCN-1100; (b) B 1s XPS peak; (c) C 1s XPS peak and (d) N 1s XPS peak; Figure S8. (a) The survey scan of XPS on BN-1200; (b) B 1s XPS peak; (c) C 1s XPS peak and (d) N 1s XPS peak; Figure S9. (a) CV curves of $\mathrm{BCN}-800, \mathrm{BCN}-900, \mathrm{BCN}-1000, \mathrm{BCN}-1100$ and $\mathrm{BN}-1200$ at a scan rate of $50 \mathrm{mV} / \mathrm{s}$; (b) discharge curves of samples obtained at different pyrolysis temperatures; Figure S10. CV curves of BCN-700 at various scan rates in $6.0 \mathrm{M}$ $\mathrm{KOH}$ electrolyte solution; Table S1: Specific surface area of samples.

Acknowledgments: This work was supported by National Natural Science Foundation of China (No. 51502098), Cultivation Plan of Outstanding youth researcher of Fujian Province and Promotion Program for Young and Middle-aged Teacher in Science and Technology Research of Huaqiao University (ZQN-PY305). The authors also appreciate helpful discussions from Jingcao Dai and Genggeng Luo.

Author Contributions: Dongping Chen conceived and designed the experiments; Dongping Chen, Yanzhen Huang, Xinling $\mathrm{Hu}$ and Rongkai Li performed the experiments together; Dongping Chen and Yingjiang Qian analyzed the data; Dongxu Li contributed reagents/materials/analysis tools; Dongping Chen wrote the manuscript.

Conflicts of Interest: The authors declare no conflict of interest.

\section{References}

1. Huang, C.J.; Chen, C.; Zhang, M.W.; Lin, L.H.; Ye, X.X.; Lin, S.; Antonietti, M.; Wang, X.C. Carbon-doped BN nanosheets for metal-free photoredox catalysis. Nat. Commun. 2015, 6, 7698-8698. [CrossRef] [PubMed]

2. Wang, S.Y.; Zhang, L.P.; Xia, Z.H.; Roy, A.; Chang, D.W.; Baek, J.; Dai, L.M. BCN Graphene as Efficient Metal-Free Electrocatalyst for the Oxygen Reduction Reaction. Angew. Chem. Int. Ed. 2012, 51, 4209-4212. [CrossRef] [PubMed]

3. Xu, Z.; Lu, W.G.; Gu, C.Z.; Liu, K.H.; Bai, X.D.; Wang, E.G.; Dai, H.J. Converting Metallic Single-Walled Carbon Nanotubes into Semiconductors by Boron/Nitrogen Co-Doping. Adv. Mater. 2008, 20, 3615-3619. [CrossRef]

4. Todi, V.O.; Shantheyanda, B.P.; Sundaram, K.B. Influence of annealing on the optical properties of reactively sputtered BCN thin films. Mater. Chem. Phys. 2013, 141, 596-601. [CrossRef]

5. Lu, J.; Li, D.X.; Yu, D.L. Study on Electronic Properties of Single-Walled $\mathrm{B}_{3} \mathrm{C}_{10} \mathrm{~N}_{3}$ Nanotubes by First Principle Calculations. Integr. Ferroelectr. 2012, 135, 17-21. [CrossRef]

6. Prakash, A.; Todi, V.; Sundaram, K.B.; Ross, L.; Xu, G.H.; French, M.; Henry, P.; King, S.W. Investigation of the Dielectric and Mechanical Properties for Magnetron Sputtered BCN Thin Films. ECS J. Solid State Sci. Technol. 2015, 4, N3122-N3126. [CrossRef]

7. Liu, X.B.; Jia, X.P.; Zhang, Z.F.; Zhao, M.; Guo, W.; Huang, G.F.; Ma, H.A. Synthesis and Characterization of New "BCN" Diamond under High Pressure and High Temperature Conditions. Cryst. Growth Des. 2011, 11, 1006-1014. [CrossRef]

8. Ma, F.K.; Wang, M.X.; Shao, Y.L.; Wang, L.J.; Wu, Y.Z.; Wang, Z.P.; Hao, X.P. 'Thermal substitution' for preparing ternary BCN nanosheets with enhanced and controllable nonlinear optical performance. J. Mater. Chem. C. 2017, 5, 2559-2565. [CrossRef]

9. Karbhal, I.; Devarapalli, R.R.; Debgupta, J.; Pillai, V.K.; Ajayan, P.M.; Shelke, M.V. Facile Green Synthesis of BCN Nanosheets as High-Performance Electrode Material for Electrochemical Energy Storage. Chem. Eur. J. 2016, 22, 7134-7140. [CrossRef] [PubMed]

10. Dou, S.; Huang, X.B.; Ma, Z.L.; Wu, J.H.; Wang, S.Y. A simple approach to the synthesis of BCN graphene with high capacitance. Nanotechnology 2015, 26, 045402. [CrossRef] [PubMed]

11. Chen, N.; Huang, X.K.; Qu, L.T. Heteroatom substituted and decorated graphene: Preparation and applications. Phys. Chem. Chem. Phys. 2015, 17, 32077-32098. [CrossRef] [PubMed]

12. Guo, H.L.; Gao, Q.M. Boron and nitrogen co-doped porous carbon and its enhanced properties as supercapacitor. J. Power. Sources 2009, 186, 551-556. [CrossRef]

13. Geick, R.; Perry, C.H.; Rupprecht, G. Normal Modes in Hexagonal Boron Nitride. Phys. Rev. 1966, 146, 543-547. [CrossRef] 
14. Li, D.X.; Lu, J.; Yu, D.L.; Tian, Y.J. Study on Raman Spectroscopy and Purification of B-C-N Compound. Metall. Mater. Trans. A 2011, 42, 2527. [CrossRef]

15. Shirai, K.; Emura, S.; Gonda, S.; Kumashiro, Y. Infrared study of amorphous $\mathrm{B}_{1-\mathrm{x}} \mathrm{C}_{\mathrm{x}}$ films. J. Appl. Phys. 1995, 78, 3392-3400. [CrossRef]

16. Lü, J.N.; Li, H.D.; Zhu, P.W.; Lü, X.Y.; Li, Y.G. Composited BCN/carbon fibers prepared by hot-filament chemical vapor deposition. Appl. Surf. Sci. 2011, 257, 4963-4967. [CrossRef]

17. Sainsbury, T.; Satti, A.; May, P.; Wang, Z.; McGovern, I.; Gun'ko, Y.K.; Coleman, J. Oxygen Radical Functionalization of Boron Nitride Nanosheets. J. Am. Chem. Soc. 2012, 134, 18758-18771. [CrossRef] [PubMed]

18. Shi, Y.; Hamsen, C.; Jia, X.; Kim, K.K.; Reina, A.; Hofmann, M.; Hsu, A.; Zhang, K.; Li, H.; Juang, Z. Synthesis of few-layer hexagonal boron nitride thin film by chemical vapor deposition. Nano Lett. 2010, 10, 4134-4139. [CrossRef] [PubMed]

19. Fei, H.L.; Ye, R.Q.; Ye, G.L.; Gong, Y.J.; Peng, Z.W.; Fan, X.J.; Samuel, E.L.G.; Ajayan, P.M.; Tour, J.M. Boron-and nitrogen-doped graphene quantum dots/graphene hybrid nanoplatelets as efficient electrocatalysts for oxygen reduction. ACS Nano 2014, 8, 10837-10843. [CrossRef] [PubMed]

20. Guo, F.S.; Yang, P.J.; Pan, Z.M.; Cao, X.N.; Xie, Z.L.; Wang, X.C. Carbon-Doped BN Nanosheets for the Oxidative Dehydrogenation of Ethylbenzene. Angew. Chem. Int. Ed. 2017, 129, 8343-8347. [CrossRef]

21. Wang, S.Y.; Iyyamperumal, E.; Roy, A.; Xue, Y.H.; Yu, D.S.; Dai, L.M. Vertically aligned BCN nanotubes as efficient metal-free electrocatalysts for the oxygen reduction reaction: A synergetic effect by co-doping with boron and nitrogen. Angew. Chem. Int. Ed. 2011, 50, 11756-11760. [CrossRef] [PubMed]

22. Weng, Q.H.; Wang, B.J.; Wang, X.B.; Hanagata, N.; Li, X.; Liu, D.Q.; Wang, X.; Jiang, X.F.; Bando, Y.; Golberg, D. Highly water-soluble, porous, and biocompatible boron nitrides for anticancer drug delivery. ACS Nano 2014, 6, 6123-6130. [CrossRef] [PubMed]

23. Zhou, J.S.; Li, N.; Gao, F.M.; Zhao, Y.F.; Hou, L.; Xu, Z.M. Vertically-aligned BCN Nanotube Arrays with Superior Performance in Electrochemical capacitors. Sci. Rep. 2014, 4, 6083-6088. [CrossRef] [PubMed]

24. Iyyamperumal, E.; Wang, S.Y.; Dai, L.M. Vertically Aligned BCN Nanotubes with High Capacitance. ACS Nano 2012, 6, 5259-5265. [CrossRef] [PubMed]

25. Zheng, L.; Wang, Z.Y.; Zhang, M.D.; Yu, C.; Wang, G.; Dong, Y.F.; Liu, S.H.; Wang, Y.W.; Qiu, J.S. Sustainable Synthesis and Assembly of Biomass-DerivedB/N Co-Doped Carbon Nanosheets with Ultrahigh Aspect Ratio for High-Performance Supercapacitors. Adv. Funct. Mater. 2016, 26, 111-119. 